\title{
Influence of thermal fluctuations on Cherenkov radiation from fluxons in dissipative Josephson systems
}

\author{
Antonov, A. A.; Pankratov, A. L.; Yulin, A. V.; Mygind, Jesper
}

Published in:

Physical Review B Condensed Matter

Link to article, DOI:

10.1103/PhysRevB.61.9809

Publication date:

2000

Document Version

Publisher's PDF, also known as Version of record

Link back to DTU Orbit

Citation (APA):

Antonov, A. A., Pankratov, A. L., Yulin, A. V., \& Mygind, J. (2000). Influence of thermal fluctuations on Cherenkov radiation from fluxons in dissipative Josephson systems. Physical Review B Condensed Matter, 61(14), 9809-9819. https://doi.org/10.1103/PhysRevB.61.9809

\section{General rights}

Copyright and moral rights for the publications made accessible in the public portal are retained by the authors and/or other copyright owners and it is a condition of accessing publications that users recognise and abide by the legal requirements associated with these rights.

- Users may download and print one copy of any publication from the public portal for the purpose of private study or research.

- You may not further distribute the material or use it for any profit-making activity or commercial gain

- You may freely distribute the URL identifying the publication in the public portal 


\title{
Influence of thermal fluctuations on Cherenkov radiation from fluxons in dissipative Josephson systems
}

\author{
A. A. Antonov, A. L. Pankratov, and A. V. Yulin \\ Institute for Physics of Microstructures of RAS, GSP 105, Nizhny Novgorod 603600, Russia \\ J. Mygind \\ Department of Physics, Technical University of Denmark, DK-2800 Lyngby, Denmark
}

(Received 2 August 1999)

\begin{abstract}
The nonlinear dynamics of fluxons in Josephson systems with dispersion and thermal fluctuations is analyzed using the "quasiparticle" approach to investigate the influence of noise on the Cherenkov radiation effect. Analytical expressions for the stationary amplitude of the emitted radiation and its spectral distribution have been obtained in an annular geometry. It is demonstrated that noise reduces the amplitude of the radiated wave and broadens its spectrum. The effect of the radiated wave on the fluxon dynamics leads to a considerably smaller linewidth than observed in the usual flux flow oscillator. A resonant behavior of both the mean amplitude and the linewidth as functions of bias current is found. The obtained results enable an optimization of the main parameters (power, tunability, and linewidth) of practical mm- and sub-mm wave Cherenkov flux flow oscillators.
\end{abstract}

\section{INTRODUCTION}

Cherenkov radiation in Josephson junctions, first suggested by Kivshar and Malomed, ${ }^{1}$ has been widely discussed during recent years. ${ }^{2-11}$ There are several motivations for the increasing interest. First, the resonant radiation emission from nonlinear systems and structures is an interesting theoretical task in it self. Second, taking into account the effect of Cherenkov radiation it is possible to explain a number of phenomena observed in experiments, e.g., microwave emission from high-temperature superconducting (HTSC) ceramics, ${ }^{9}$ and fine structures in the current-voltage characteristics (IVC's) of annular Josephson junctions. ${ }^{11}$ Finally, and important for applications, the tunable resonant interaction of Josephson fluxons with electromagnetic waves allows for a significant increase of the efficiency of Josephson oscillators based on fluxon dynamics. In addition to the increased emitted mm- and sub-mm wave power, the resonant nature of the Cherenkov effect simultaneously allows for a significant reduction of the linewidth and the content of higher harmonics. Compared to the standard flux flow oscillator (FFO) (Ref. 10) and the Josephson soliton oscillator (JSO) (Ref. 12) where the power is emitted only from the end(s) of a one-dimensional long Josephson junction, the Cherenkov flux flow oscillator (CFFO) extracts energy from the whole fluxon chain as it propagates through the junction. The CFFO therefore may be considered as a new type of Josephson oscillator.

The influence of noise on the Cherenkov radiation effect has not yet been practically investigated. The results obtained for the JSO, where the radiation linewidth is mainly determined by fluctuations in the velocity of fluxons, freely moving in a long Josephson junction, ${ }^{13}$ cannot be directly applied to the CFFO. For the FFO extensive measurements of the linewidth have been made but no reliable noise theory has yet been proposed. Recently it has been shown that the FFO can be phase locked to high harmonics of an external reference oscillator and tuned in-lock over a wide frequency band (270-440 GHz). ${ }^{14,15}$ In the CFFO where fluxons are moving in the periodic potential created by the accumulated radiated wave generated by the fluxons themselves the problem becomes even more nonlinear, and at the present time there exists no elaborated theory for the linewidth. The influence of noise on power and radiation linewidth is important both from fundamental and practical points of view, and the aim of this paper is to present a simple but sufficiently precise theory that enables a reliable estimation of the basic parameters needed for the practical design of the CFFO.

\section{THE BASIC PROBLEM}

The physical system considered is a one-dimensional long Josephson junction (LJJ) coupled to a linear external transmission line, e.g., a waveguide. The normalized junction length $l$ and width $w$ satisfy $l \gg 1$ and $w \ll 1$; normalization is to $\lambda_{J}$, the Josephson penetration depth. In a long junction magnetic flux is quantized and enters in the form of fluxons (Josephson vortices), each containing a single flux quantum $\Phi_{0}=h /(2 e)$. Subjected to suitable bias conditions, usually an applied dc bias current and magnetic field, a fluxon moves along the junction as a solitary wave which in many respects behaves as a (quasi)particle. Cherenkov radiation exists when the particle velocity exceeds (or equals) the phase velocity of the wave it generates. The type of radiation and its phase velocity depends on the surrounding medium.

In our system an external transmission line is the medium which provides the "slow" waves that can be in resonance with the moving fluxons in order to satisfy the Cherenkov condition. The Josephson junction, which in itself is a transmission line, cannot be used because the phase velocity (Swihart velocity) of its linear modes always is larger than the maximal fluxon velocity. There are several ways the waves in the external transmission line may appear slow relative to the fluxon. The simplest coupling scheme we can 


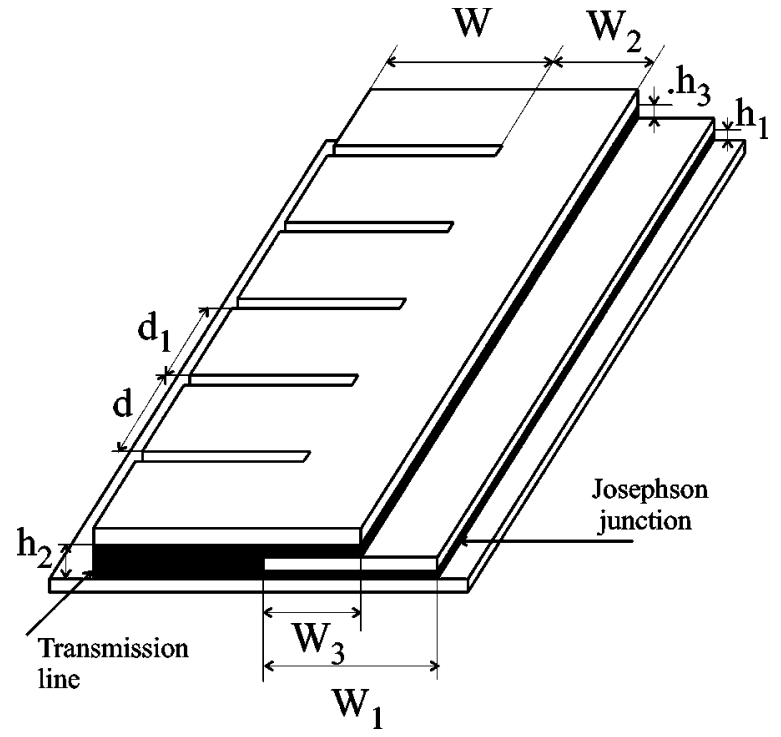

FIG. 1. Sketch of a section of a long Josephson junction coupled to a slow wave transmission line with dispersion (see text). The structure is one of the possible schemes realizing the Cherenkov flux flow oscillator (CFFO).

propose is to overlay the junction with a meander shaped stripline that crosses (and couples) to the junction at regularly spaced intersections. The wave generated in the stripline by a fluxon at a given intersection must travel a long distance in the side arm of the meander (and therefore is considerably delayed) before it again meets the fluxon at the next intersection. If the coupling between the junction and the stripline is weak the propagating wave on the external transmission line obeys a simple wave equation containing a linear operator (see below).

The coupling scheme suggested above is an example of the general distributed system of Josephson junctions electromagnetically coupled to a wave guide. A distributed coupling scheme similar to that shown in Fig. 1 for a LJJ and the corresponding discrete electronic circuit model has been treated theoretically by Kurin and Yulin. ${ }^{16}$ They show that the fluxons bunch in the decelerating phase of the field propagating in the waveguide in a process that closely mimics the way electrons bunch in the traveling-wave and backward wave tubes. If the wave guide system is periodic (e.g., contains resonant sections and thus appears dispersive) one may, for simplicity, treat the system in the long wave limit assuming that its spatial period is much smaller than the wavelength of the excited wave in the waveguide. This avoids the additional difficulties with finding the necessary eigenfunctions and the dispersion relation. The general case, where the slow wave also can be provided but where all the coefficients in the equations describing the system depend on the space coordinate can in principle be solved. This, however, leads to very long calculations.

Recently Baryshev et al. ${ }^{17}$ proposed an alternative slow wave structure consisting of a (main) transmission line (waveguide) with periodically spaced resonant side arms each of which are end coupled to the side of the LJJ. The delay of the excited wave in the side arms makes the returning wave to appear slow to the fluxons while the excited waves in all side arms interfere constructively and propagate in the main line. The waveguide system is spatially periodic but its wave equation can still be described by a linear operator. The dispersion characteristic of the linear waves exhibits a zone structure and the required resonance condition can be achieved in a sufficiently high numbered Brillouin zone (resonance between a fluxon and high space harmonic of the eigenfunction of the linearized operator).

In order to find analytical solutions for the CFFO with noise we will consider the simplest case, which is the annular system, i.e., a long annular junction coupled to an overlaying annular transmission line, e.g., a microstrip line. A short section of the structure is shown in Fig. 1. In the linear geometry the interaction of fluxons with the LJJ boundaries, and collisions of fluxons and antifluxons can only be dealt with using numerical simulations. We admit that the annular system may appear somewhat academic, but we can use it to demonstrate the most important features and effects. Nevertheless, the annular system can be realized in practice ${ }^{11,18}$ and what is very important is that the mm- or sub-mm wave power generated by the Cherenkov process can be coupled out using either the meander or the resonant antenna/ waveguide systems suggested above. Both the linear and the annular geometry appear suitable for practical CFFO applications.

Due to flux quantization the number of fluxons trapped in an annular junction is constant, depending only on how the system was prepared. For the current biased annular Josephson junction the well known sine-Gordon equation can be written

$$
\begin{aligned}
\varphi_{t t}+ & \alpha \varphi_{t}-\varphi_{x x}+\sin \left(\varphi+\frac{2 \pi N x}{l}\right) \\
& =-\gamma \psi_{x x}+\beta \psi_{t t}+\eta_{0}+\zeta(x, t) .
\end{aligned}
$$

The transmission line is described by the equation

$$
\hat{D} \psi=-\gamma \varphi_{x x}+\beta \varphi_{t t} .
$$

Here $\varphi+2 \pi N x / l, \alpha, \eta_{0}, \zeta$ are, respectively, the phase difference of the order parameter of the Josephson junction, the damping coefficient of the junction, the bias current, and the noise current. We assume that the current density is constant and that the dc bias current is supplied uniformly along the junction. All currents are normalized to the critical current. $N$ is the number of fluxons trapped in the junction, and $\gamma$ and $\beta$ are dimensionless coefficients describing the coupling between the fluxon and the transmission line, see Refs. 19,20. Note that an annular junction can only be locally described by the differential equation for the Josephson phase difference in the case when there is magnetic flux in the junction. The reason is that in this case $\varphi_{J}$ changes with $2 \pi N$ when one follows a closed pass along the junction. So this variable is not differentiable in any point of the junction. Instead we use in our equations the variable $\varphi=\varphi_{J}-2 \pi N x / l$ which is well defined in the system. The linear integrodifferential operator $\hat{D}$ determines the dispersion properties of the external waveguide system which allows for the Cherenkov synchronism. For example for the system shown on the Fig. 1 the appearance of $\hat{D}$ in the long wave approximation is the following: 


$$
\begin{aligned}
D(\omega, k)= & -\omega^{2} \frac{\epsilon_{2}}{\epsilon_{1}} \frac{h_{1}}{W_{1}}\left(\frac{W_{2}}{h_{2}}+\frac{d_{1}}{d} \frac{W}{h_{2}} \frac{\tan (\omega W / v)}{\omega W / v}\right) \\
& +k^{2} \frac{\mu_{1}}{\mu_{2}} \frac{h_{1}+2 \lambda}{W_{1}} \frac{W_{2}}{h_{2}+2 \lambda},
\end{aligned}
$$

in the $\omega-k$ representation, see Ref. 16. Here $h_{1}, h_{2}, W, W_{1}, W_{2}, d, d_{1}$ are geometrical parameters of the Josephson junction and the transmission line, see Fig. 1, $\epsilon_{1}, \mu_{1}, \epsilon_{2}$ and $\mu_{2}$ are dielectric and magnetic constants of the Josephson junction and the transmission line, $\lambda$ is London penetration depth for the superconducting electrodes, $v$ $=\sqrt{\epsilon_{1} \mu_{1} / \epsilon_{2} \mu_{2}} \sqrt{h_{2} /\left(h_{2}+2 \lambda\right) /\left(h_{1}+2 \lambda\right)}$ is the velocity of the light in the transmission line. It is seen that at $\omega \rightarrow 0$ the expression (3) becomes the usual D'Alambert operator. But the impedance of the side outgrowths of the transmission line is a function of frequency and so they can work as inductors as well as capacitors depending on the frequency. It is accounted by the term $\tan (\omega W / v) / \omega W / v$ in the expression for $\hat{D}$.

Equations (1),(2) differ from the equations considered in Ref. 16 only by the noise current $\zeta(x, t)$ introduced on the right-hand side of Eq. (1). Note here that in general we have to take into account also the thermal noise in the transmission line. But this noise is weak in terms of its influence on Josephson soliton dynamics (not only because these fluctuations have small intensity but also because the coupling between the Josephson junction and the transmission line is supposed to be weak). So the thermal noise in the transmission line only leads to a small pedestal in the radiation spectrum but not to a real broadening of the radiation linewidth.

Dimensionless variables are used throughout this paper, with time measured in units of the maximum inverse Josephson plasma frequency $\left(1 / \omega_{p}\right)$ and length in units of the Josephson penetration length $\left(\lambda_{J}\right)$. This implies that velocities are in units of the Swihart velocity $\left(c_{s}=\lambda_{J} \omega_{p}\right)$. Subindex $x$ and $t$ indicates spatial and temporal derivative, respectively.

A white Gaussian noise current is assumed

$$
\begin{gathered}
\langle\zeta(x, t)\rangle=0 \\
\left\langle\zeta\left(x_{1}, t_{1}\right) \zeta\left(x_{2}, t_{2}\right)\right\rangle=\frac{16 k_{B} T \alpha}{E_{0}} \cdot \delta\left(t_{1}-t_{2}\right) \cdot \delta\left(x_{1}-x_{2}\right),
\end{gathered}
$$

where $T$ is the temperature, $k_{B}$ is Boltzmann's constant, and $E_{0}$ is the rest energy of the fluxon.

Equations (1) and (2) have to be supplemented with proper boundary conditions. For the annular junction the boundary conditions can be written as

$$
\varphi(x)=\varphi(x+l), \quad \psi(x)=\psi(x+l) .
$$

In our model the transmission line and the junction have the same length $l$, e.g., they are placed directly on top of each other. This is not a major restriction, but it helps not to complicate the calculations unnecessarily. In the annular geometry (and even with a meanderlike transmission line) one could have used an angular parameter (or $x$ along the unfolded meander) to characterize position on both the junction and the transmission line.
Before we discuss the technical aspects of the noise problem it is appropriate to formulate our final goal which is to derive a self-consistent system of abridged equations that describe the Josephson system in terms of the fluxon movement and the transmission line in terms of a slowly varying complex amplitude of the radiated wave. The first step is to introduce the quasiparticle approach for the junction.

When the terms in Eq. (1) corresponding to damping, bias current and the backward action of the electrodynamic system are small, the solitary wave solution to the nearly unperturbed sine-Gordon equation dynamically behaves as a quasiparticle which may be assigned an effective mass, momentum, kinetic energy, etc. In this quasiparticle approximation, and when the distance between successive fluxons is much larger than the fluxon length, the solution to Eq. (1) may be presented as a sum of solitary solutions to the unperturbed sine-Gordon equation and a small nongrowing component $\varphi=\sum_{n=1}^{N} \varphi_{s n}+\lambda$. The solitary solution to Eq. (1) is well known,

$$
\begin{aligned}
\varphi_{s n}(x, t) & =f_{\text {sol }}\left[x-X_{n}(t)\right] \\
& = \pm 4 \arctan \exp \left(\frac{x-X_{n}}{\sqrt{1-\dot{X}_{n}^{2}}}\right)-\frac{2 \pi x}{l} .
\end{aligned}
$$

Let us consider the case when the direction of the fluxon magnetic field corresponds to a minus sign in front of the arctan in Eq. (5). Following Ref. 12 one gets the following equation for the center $X_{n}$ of the $n$th fluxon:

$$
\begin{aligned}
\ddot{X}_{n}+\alpha \dot{X}_{n}= & \sqrt{1-\dot{X}_{n}^{2}}\left(\frac{\pi}{4} \eta_{0}-\frac{1}{8} \int_{-\infty}^{\infty}\left[\zeta(x, t)-\gamma \psi_{x x}\right.\right. \\
& \left.\left.+\beta \psi_{t t}\right] f_{\mathrm{sol}}^{\prime}\left[x-X_{n}(t)\right] d x\right) .
\end{aligned}
$$

We have neglected the fluxon-fluxon interaction which is correct when the distance between neighbor fluxons is much greater than their size. This condition probably will be fulfilled in the beginning of the Cherenkov generation process. Later in the stationary resonant state the interaction with the wave leads to the situation where each fluxon is located near a minimum of the electromagnetic wave, so that the fluxonfluxon distance does not decrease. Inclusion of the fluxonfluxon interaction requires numerical simulation.

In the noise-free case without coupling to the transmission line $(\gamma=\beta=0, \zeta=0)$ the fluxons move uniformly as a "diluted" chain with the normalized velocity $V_{a}\left(\eta_{0}\right)$ determined by the power balance condition

$$
\frac{\alpha V_{a}}{\sqrt{1-V_{a}^{2}}}=\frac{\pi}{4} \eta_{0}
$$

which can be easely derived from either Eq. (6), or, with more calculations, directly from Eq. (1).

Since the system is annular, the field in the stripline waveguide can be represented in the following form:

$$
\psi(x, t)=\sum_{m=0}^{\infty} b_{m}(t) e^{-i k_{m} x}+\text { c.c. }
$$


where $k_{m}=2 \pi m / l$ is the normalized wave vector of the $m$ th mode with amplitude $b_{m}(t)$. The most effective excitation will take place for the wave running with a phase velocity close to the fluxon velocity, in other words for the wave which is in Cherenkov synchronism with the fluxons. The condition of this synchronism is $\omega / k=V_{r}$ where $V_{r}$ is the normalized fluxon velocity at resonance. This resonance interaction will be considered below subjected to thermal noise.

Let only one mode with wave number $k_{r}$ close to the synchronism point be excited. This takes place when the distances between modes $\Delta \omega=V_{g}\left(k_{r}\right) 2 \pi / l$ are much larger than the dissipation constant $\Gamma$

$$
V_{g}\left(k_{r}\right) \frac{2 \pi}{l} \gg \Gamma,
$$

where $\Gamma$ is the mode damping rate and $V_{g}$ is the group velocity of the transmission line.

Then the field in the electromagnetic system may be approximated by

$$
\begin{gathered}
\psi(x, t)=a(t) \cdot \exp \left(i \omega_{r} t-i k_{r} x\right)+\text { c.c. }, \\
a(t)=A(t) \exp [i \chi(t)] .
\end{gathered}
$$

Here $a(t), A(t)$, and $\chi(t)$ are slowly varying functions of time on the time scale of $1 / \omega_{r}$. The resonance frequency $\omega_{0}=\omega_{r}+i \Gamma$ of the mode is determined from $D\left(\omega_{r}\right.$ $\left.+i \Gamma, k_{r}\right)=0$, where

$$
D(\omega, k)=\frac{1}{4 \pi^{2}} \int_{-\infty}^{+\infty} \int_{-\infty}^{+\infty} \hat{D}(t, x) e^{-i \omega t+i k x} d t d x
$$

is the Fourier image of the dispersion operator $\hat{D}$. Note here, that the complex amplitude $a$ has the characteristic time scale of variation that equals $\Gamma^{-1}=\left[\operatorname{Im}\left(\omega_{0}\right)\right]^{-1}$, where $\Gamma \ll \omega_{r}$. We also have assumed that the offset of the fluxon velocity from the phase velocity of the electromagnetic wave $V_{r}$ $=\omega_{r} / k_{r}$ is so small that

$$
\left|\dot{X}_{n}-V_{r}\right| \ll\left(1-V_{r}^{2}\right) / V_{r},
$$

meaning that we can neglect changes in the shape of the fluxon.

Applying the method of slowly varying amplitudes let us proceed with the abridged equations for the complex amplitude $a$. Taking the Fourier transform of left and right parts of Eq. (2) where $\varphi$ is a series of single soliton solutions [Eq. (5)] one can obtain the equation

$$
\hat{D}\left(t, k_{r}\right) a(t) e^{i \omega_{r} t}=-\frac{i \sigma_{0}}{l} \sum_{n=1}^{N} e^{i k_{r} X_{n}},
$$

where $\sigma_{0}=k_{r}\left(\beta V_{r}^{2}-\gamma\right) / \cosh \left[(\pi / 2) k_{r} \sqrt{1-V_{r}^{2}}\right]$. Now let us write out the representation of $\hat{D}\left(t, k_{r}\right)$ through $D\left(\omega, k_{r}\right)$

$$
\hat{D}\left(t, k_{r}\right) a(t) e^{i \omega_{r} t}=\int_{-\infty}^{\infty} D\left(\omega, k_{r}\right) a\left(\omega-\omega_{r}\right) e^{i \omega t} d \omega .
$$

Since $a(t)$ is a slow function of time it is possible to substitute $D\left(\omega_{r}+i \Gamma, k_{r}\right)+(\partial D / \partial \omega)\left(\omega-\omega_{r}-i \Gamma\right)$ into Eq. (9) in- stead of $D\left(\omega, k_{r}\right)$. Taking into account that $D\left(\omega_{r}+i \Gamma, k_{r}\right)$ $=0$ after some simplification we have

$$
\begin{aligned}
\hat{D}\left(t, k_{r}\right) a(t) e^{i \omega_{r} t} & =e^{i \omega_{r} t} \frac{\partial D}{\partial \omega} \int_{-\infty}^{\infty}(\delta-i \Gamma) a(\delta) e^{i \delta t} d \delta \\
& =e^{i \omega_{r} t} \frac{\partial D}{\partial \omega}\left(-i \frac{\partial a}{\partial t}-i \Gamma a\right) .
\end{aligned}
$$

And then, finally, we obtain the abriged equation for $a(t)$ in the form

$$
\begin{gathered}
\dot{a}+\Gamma a=\epsilon \sum_{n=1}^{N} e^{-i \omega_{r} t+i k_{r} X_{n},} \\
\epsilon=\frac{\sigma_{0}}{l}\left(\left.\frac{\partial D\left(\omega, k_{r}\right)}{\partial \omega}\right|_{\omega_{0}}\right)^{-1},
\end{gathered}
$$

and the quasiparticle equations for the fluxons, Eq. (6), where $\psi(x, t)=a(t) \exp \left(i \omega_{r} t-i k_{r} x\right)$ has to be substituted in $\psi_{x x}$ and $\psi_{t t}$.

These equations can be cast in a more suitable form. Let us write the equation for the wave amplitude in real (not complex) variables $A$ and $\chi$. Writing out real and imaginary parts of the Eq. (10) we obtain

$$
\begin{gathered}
\dot{A}+\Gamma A=\epsilon \sum_{n=1}^{N} \sin \theta_{n}, \\
\dot{\chi} A=-\epsilon \sum_{n=1}^{N} \cos \theta_{n},
\end{gathered}
$$

where

$$
\theta_{n}=k_{r} X_{n}-\omega_{r} t-\chi(t)-\pi / 2 .
$$

Note here that the fluxons can be described by their phases. It is more convenient because this variable in particular characterizes their interaction with the wave. The phase coordinate $\theta_{n}$ determines the fluxon location with respect to the wave and may be used instead of the linear coordinate $X_{n}$. The time derivative of the phase $\chi(t)$ represents the deviation of the frequency of the emitted wave from the resonance frequency $\omega_{r}$. In the new variables $\theta_{n}, A, \chi$ the equation for the center of a fluxon takes the form

$$
\begin{aligned}
\left(\ddot{\theta}_{n}+\ddot{\chi}\right)+\alpha_{1}\left(\dot{\theta}_{n}+\dot{\chi}\right)= & \alpha_{1} \sigma_{1}\left(\eta_{0}-\eta_{r}\right)-\sigma A \sin \theta_{n} \\
& +\alpha_{1} \eta_{f n}(t),
\end{aligned}
$$

where the normalized resonance bias current, $\eta_{r}$, is determined by $V_{a}\left(\eta_{r}\right)=V_{r}$;

$$
\sigma=\frac{\pi \sigma_{0}}{2} k_{r}^{2} \sqrt{1-V_{r}^{2}}, \quad \sigma_{1}=\frac{\pi}{4 \alpha_{1}} k_{r} \sqrt{1-V_{r}^{2}}, \quad \alpha_{1}=\frac{\alpha}{1-V_{r}^{2}} .
$$

The random force $\eta_{f n}(t)$ is a $\delta$-correlated Gaussian process

$$
\begin{gathered}
\left\langle\eta_{f n}(t)\right\rangle=0,\left\langle\eta_{f n}\left(t_{1}\right) \eta_{f k}\left(t_{2}\right)\right\rangle=2 D \delta_{n, k} \delta\left(t_{1}-t_{2}\right), \\
D=\frac{8 k T\left(1-V_{r}^{2}\right)^{3 / 2} k_{r}^{2}}{\alpha_{1} E_{0}} .
\end{gathered}
$$


We now have a system of equations for the real amplitude, Eq. (12), the phase of the wave, Eq. (13), and the phase coordinate $\theta_{n}$, Eq. (15), but unfortunately it is still too complicated to be solved analytically. In order to proceed we consider a special case which on one hand is quite realistic but on the other hand allows for analytical solutions.

When the fluctuations are small the following conditions are fulfilled $\langle A\rangle \gg \sqrt{\left\langle A^{2}\right\rangle-\langle A\rangle^{2}},\langle\dot{\chi}\rangle \gg \sqrt{\left\langle\dot{\chi}^{2}\right\rangle-\langle\dot{\chi}\rangle^{2}}$. This assumption gives the possibility to neglect the fluctuations of both the amplitude and the frequency in Eq. (15) and substitute $\langle A\rangle$ for $A$ and $\langle\dot{\chi}\rangle$ for $\dot{\chi}$. All equations will be considered in the stationary limit when the steady-state values of the amplitude and the frequency have been reached. This condition together with the previous one $\langle\dot{\chi}\rangle$ $\gg \sqrt{\left\langle\dot{\chi}^{2}\right\rangle-\langle\dot{\chi}\rangle^{2}}$ allows us to neglect also the $\ddot{\chi}$ term. In other words we neglect the influence of the fluctuations of the wave amplitude and the frequency on the dynamics of the fluxon phase. The justification for this procedure will be given later when we have found the expressions for the spectra of $A$ and $\dot{\chi}$.

In the stationary limit we can rewrite Eq. (15) as

$$
\begin{aligned}
\ddot{\theta}_{n}+\alpha_{1} \dot{\theta}_{n}= & \alpha_{1} \sigma_{1}\left(\eta_{0}-\eta_{r}\right)-\alpha_{1}\langle\dot{\chi}\rangle-\sigma\langle A\rangle \sin \theta_{n} \\
& +\alpha_{1} \eta_{f n}(t) .
\end{aligned}
$$

Equation (18) only depends on one variable, $\theta_{n}$, and can be investigated analytically. After simplifications of Eqs. (12), (13), and (15) we actually have two linear equations (12) and (13) with some random process (noise) on the right-hand sides $\left(\sum_{n=1}^{N} \sin \theta_{n}\right.$ and $\left.\sum_{n=1}^{N} \cos \theta_{n}\right)$. These random processes have complicated statistical properties that can be found from Eq. (18).

Equation (18) gives us the statistics for $\theta_{n}$ which certainly depends on $\langle\dot{\chi}\rangle$ and $\langle A\rangle$. On the other hand, knowing the statistics of $\theta_{n}$ we can find $\langle\dot{\chi}\rangle$ and $\langle A\rangle$. So, we have a set of transcendental equations (12)-(18) for $\langle\dot{\chi}\rangle$ and $\langle A\rangle$. Solving this system numerically we will be able to find the mean amplitude and the frequency deviation. Having done that we can finally investigate the spectral characteristics of the excited wave. Below we will make the first step and investigate the statistical properties of $\theta_{n}$.

Equation (18) is still rather complicated, but for many real cases we can assume that $\alpha_{1}^{2} \gg \sigma\langle A\rangle$. Indeed, the maximal amplitude $\langle A\rangle$ is of the order of $\langle A\rangle \approx \epsilon N / \Gamma$ (see Ref. 16) and this condition takes the form $\alpha_{1}^{2} \Gamma \gg \sigma \epsilon N$. This can be satisfied in a wide range of parameters. Notice, that the last condition does not imply that the junction is overdamped. It has been demonstrated ${ }^{21}$ (see also Ref. 22), that the condition $\alpha_{1}^{2} \gg \sigma\langle A\rangle$ implies that the second time derivative of $\theta_{n}$ in Eq. (18) may be neglected.

Let us renormalize the amplitude $A^{\prime}(t)$ so that the optimal amplitude, reached in the absence of noise, is equal to unity; $A^{\prime}(t)=A(t) \Gamma / \epsilon N$. Finally we get the following equations for $A^{\prime}(t), \dot{\chi}(t)$, and $\theta_{n}(t)$ :

$$
\dot{\theta}_{n}=\frac{1}{h}\left[\xi_{d}-h\langle\dot{\chi}\rangle-\left\langle A^{\prime}\right\rangle \sin \left(\theta_{n}\right)\right]+\eta_{f n}(t)
$$

$$
\begin{gathered}
\frac{1}{\Gamma} \dot{A}^{\prime}(t)+A^{\prime}(t)=\frac{1}{N} \sum_{n=1}^{N} \sin \left[\theta_{n}(t)\right], \\
\dot{\chi}=-\frac{\Gamma}{A^{\prime} N} \sum_{n=1}^{N} \cos \left[\theta_{n}(t)\right],
\end{gathered}
$$

where $h=\Gamma \alpha_{1} / \sigma \epsilon N$ includes the effective mode damping rate $\Gamma$ and $\xi_{d}=h \sigma_{1}\left(\eta_{0}-\eta_{r}\right)$ is an effective "current" difference between the dc bias current and the normalized current at resonance. The Langevin equation, Eq. (19), is completely analogous to the corresponding equation that describes the dynamics of a single overdamped Josephson junction.

\section{MEAN AMPLITUDE OF CHERENKOV RADIATION AND CURRENT-VOLTAGE CHARACTERISTIC}

In order to obtain the averages of $A^{\prime}$ and $\dot{\chi}$, namely their mean and correlation functions (spectra), it is necessary to know the corresponding averages of $\sin \left[\theta_{n}(t)\right]$ and $\cos \left[\theta_{n}(t)\right]$ where the phase $\theta_{n}(t)$ is a stochastic variable governed by the Langevin equation, Eq. (19). All fluxons have the same statistical properties, i.e., the random process $\theta_{n}(t)$ does not depend on the index $n$. In this and the next section we denote $\theta_{n}(t)=\theta(t)$. The necessary averages of the phase $\theta$ may be obtained using the Fokker-Planck equation (FPE) for the probability density $W(\theta, t)$ corresponding to Eq. (19)

$$
\begin{aligned}
\frac{\partial W(\theta, t)}{\partial t} & =-\frac{\partial G(\theta, t)}{\partial \theta} \\
& =D \frac{\partial}{\partial \theta}\left\{\left[\frac{d u(\theta)}{d \theta} W(\theta, t)\right]+\frac{\partial W(\theta, t)}{\partial \theta}\right\},
\end{aligned}
$$

where $G(\theta, t)$ is the probability current and $u(\theta)$ is the potential

$$
u(\theta)=-\left[\left\langle A^{\prime}\right\rangle \cos (\theta)+\Delta \eta \theta\right] / h D, \Delta \eta=\xi_{d}-h\langle\dot{\chi}\rangle .
$$

The initial and boundary conditions for Eq. (22) with the potential Eq. (23) are

$$
W(\theta, 0)=\delta\left(\theta-\theta_{0}\right) \text { and } W(\pi, t)=W(-\pi, t) .
$$

The mean steady-state amplitude and the mean frequency shift may be found using only the stationary solution to the FPE (see below). In order to obtain the correlation functions it is necessary to know the nonstationary solution of the FPE, Eq. (22), but unfortunately this is not available. However, the problem of obtaining the nonstationary solution may be avoided using the original approach presented in Sec. IV.

Here we introduce another simplification that will be used in the analysis of Eq. (21). In this equation we have a quotient of two random processes; $\sum_{n=1}^{N} \cos \left[\theta_{n}(t)\right]$ and $A^{\prime}(t)$. Within the frame of our previous restriction $\langle A\rangle$ $\gg \sqrt{\left\langle A^{2}\right\rangle-\langle A\rangle^{2}}$ we can present $A^{\prime}$ as $A^{\prime}=\left\langle A^{\prime}\right\rangle+A_{f}^{\prime}$, decompose the denominator of Eq. (21) to the set, and neglect the small fluctuations of the amplitude $A_{f}^{\prime}$ in comparison with $\left\langle A^{\prime}\right\rangle$. Accordingly, in the equation for $\dot{\chi}$ we will always suppose $A^{\prime} \approx\left\langle A^{\prime}\right\rangle$. This procedure does not give additional 
limitations because we already have assumed that the amplitude variation near its mean value is small.

When averaging Eq. (20) in the stationary case $\left\langle\dot{A}^{\prime}\right\rangle=0$ we get

$$
\left\langle A^{\prime}\right\rangle=\langle\sin (\theta)\rangle,
$$

utilizing $\sum_{n=1}^{N}\left\langle\sin \left(\theta_{n}\right)\right\rangle=N\left\langle\sin \left(\theta_{n}\right)\right\rangle=N\langle\sin (\theta)\rangle$.

The average $\langle\sin \theta\rangle=\int_{-\infty}^{\infty} \sin (x) W(x, t) d x$ may be obtained using the approach of reduced phases. ${ }^{23,24}$ If free (natural) boundary conditions were used for the diffusion in the potential Eq. (23) then the steady-state solution of the FPE will be zero; $W_{s t}(\theta)=W(\theta, \infty)=0$. Because of the periodicity of the function $\sin (\theta)$ one can introduce periodic boundary conditions, Eq. (24), and consider the process of diffusion within the reduced interval $(-\pi, \pi)$ due to the fact that

$$
\begin{aligned}
\int_{-\infty}^{\infty} \sin (x) W(x, t) d x= & \sum_{n=-\infty}^{\infty} \int_{-\pi}^{\pi} \sin (x+2 \pi n) \\
& \times W(x+2 \pi n, t) d x \\
& =\int_{-\pi}^{\pi} \sin (x) W_{r}(x, t) d x
\end{aligned}
$$

where $W_{r}(x, t)$ is the reduced probability density. This reduced probability density will reach a nonzero steady-state distribution for $t \rightarrow \infty$. The steady-state reduced probability density $W_{r}(\theta, \infty)=W_{r s t}(\theta)$ may be obtained from Eq. (22), supposing that the time derivative is equal to zero, and integrating the reminder parts twice. The two arbitrary constants are determined from the periodic boundary condition, Eq. (24), and from the normalization condition $\int_{-\pi}^{\pi} W_{r s t}(\theta) d x$ $=1$. The reduced steady-state probability density $W_{r s t}(\theta)$ has the form

$$
W_{r s t}(\theta)=\frac{e^{-u(x)} \int_{x}^{x+2 \pi} e^{u(y)} d y}{\int_{-\pi}^{\pi} e^{-u(x)} \int_{x}^{x+2 \pi} e^{u(y)} d y d x},
$$

where the potential $u(x)$ is given in Eq. (23). Using the obtained reduced steady-state probability density one gets $\langle\sin (\theta)\rangle$ and substituting it into Eq. (25) one can write the following transcendental equation for the mean steady-state amplitude:

$$
\left\langle A^{\prime}\right\rangle=\frac{\int_{-\pi}^{\pi} \sin (x) e^{-u(x)} \int_{x}^{x+2 \pi} e^{u(y)} d y d x}{\int_{-\pi}^{\pi} e^{-u(x)} \int_{x}^{x+2 \pi} e^{u(y)} d y d x},
$$

where $u(x)$ [Eq. (23)] is a function of $\left\langle A^{\prime}\right\rangle$.

A similar procedure may be used to obtain the transcendental equation for $\langle\dot{\chi}\rangle$

$$
\langle\dot{\chi}\rangle=-\frac{\Gamma}{\left\langle A^{\prime}\right\rangle} \frac{\int_{-\pi}^{\pi} \cos (x) e^{-u(x)} \int_{x}^{x+2 \pi} e^{u(y)} d y d x}{\int_{-\pi}^{\pi} e^{-u(x)} \int_{x}^{x+2 \pi} e^{u(y)} d y d x},
$$

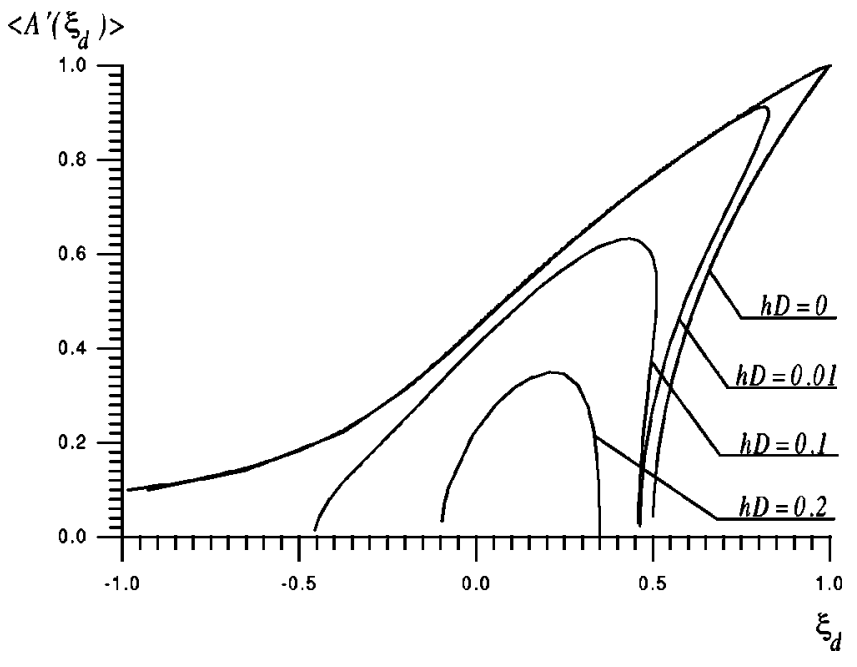

FIG. 2. Calculated dimensionless mean amplitude $\left\langle A^{\prime}\right\rangle$ of the radiation generated in an annular CFFO as function of bias current for different values of the noise intensity $h D$ and fixed effective mode damping rate $h \Gamma=0.1$. The zero on the horizontal axis is the Cherenkov synchronism resonance current. All parameters are normalized, see text.

where $\left\langle A^{\prime}\right\rangle$ should be substituted as a solution to Eq. (27). The system of transcendental equations Eq. (27) and Eq. (28) can be solved numerically for any given value of $\Delta \eta=\xi_{d}$ $-h\langle\dot{\chi}\rangle$ and the mean value of the amplitude may be plotted as function of $\xi_{d}$, the shifted normalized bias current. When noise is absent $(D=0)$ the formula for the amplitude is $A^{\prime}$ $=\sqrt{\Delta \eta}$ (stable branch) or $A^{\prime}=\sqrt{2 \Delta \eta-1}$ (unstable branch). In this case the point $\xi_{d}=1(\Delta \eta=1)$ corresponds to the upper limit of the stable regime of the Cherenkov radiation. This point also is the point of maximal amplitude.

Figure 2 shows plots of the normalized mean amplitude $\left\langle A^{\prime}\right\rangle$ as function of $\xi_{d}$ for different values of the noise intensity, $h D$. As demonstrated in Fig. 2 when the noise intensity increases, the region of bias current where the Cherenkov radiation exists becomes smaller and the bias current corresponding to the maximal amplitude decreases, moving to the middle of the generation range. All curves in Fig. 2 are calculated for a fixed value of the effective mode damping parameter, $h \Gamma=0.1$.

The current-voltage characteristic (IVC) may be easily obtained from the data calculated for the mean amplitude $\left\langle A^{\prime}\right\rangle$. The normalized mean voltage drop is $\langle V\rangle=\left\langle\dot{X}_{n}\right\rangle$ where $X_{n}$ is the coordinate of the center of the fluxon. Expressing $X_{n}$ from formula Eq. (14) and taking $\left\langle\theta_{n}\right\rangle$ from Eq. (19) one finds the following formula for the normalized mean voltage as function of the bias current:

$$
\langle V\rangle=\frac{\omega_{r}}{k_{r}}+\frac{1}{h k_{r}}\left(\xi_{d}-\left\langle A^{\prime}\right\rangle^{2}\right) .
$$

Figures 3 and 4 shows IVCs, $U\left(\xi_{d}\right)=\left\langle V\left(\xi_{d}\right)\right\rangle-\omega_{r} / k_{r}$, calculated for different values of the noise intensity with $h \Gamma=0.01$ and $h \Gamma=1$, respectively. The horizontal "current" variable is $\xi_{d}=h \sigma_{1}\left(\eta_{0}-\eta_{r}\right)$ containing the difference between the normalized dc bias current and the current at resonance. It is seen, that the hysteresis increases for smaller values of the mode damping rate $\Gamma$. On the other hand, an 


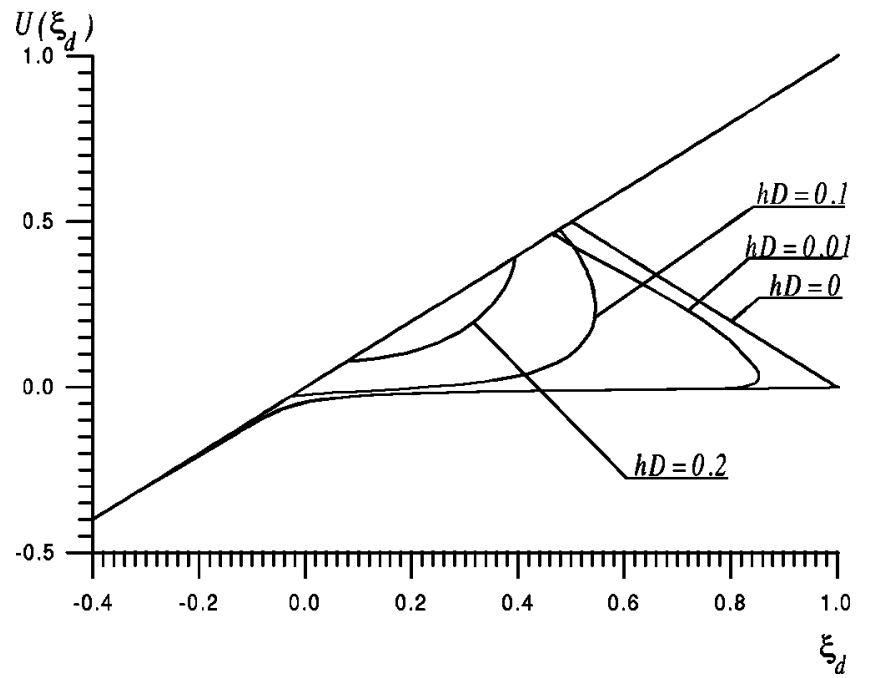

FIG. 3. Current-voltage characteristic calculated for different values of the noise intensity $h D$ with fixed $h \Gamma=0.01$. The zero on the horizontal axis is the Cherenkov synchronism resonance current. All parameters are normalized, see text.

increase of either the noise intensity $D$ or $h$ (e.g., decreasing the coupling coefficients) reduces the hysteresis.

\section{CORRELATION FUNCTIONS AND SPECTRA OF AMPLITUDE AND FREQUENCY OF CHERENKOV RADIATION}

The equation for the correlation function of the amplitude may easily be obtained from Eq. (20). Consider Eq. (20) at time $t_{1}$. Multiplying it by the same equation at time $t_{2}$ and averaging one gets the equation for the correlation function $K_{A}\left[t_{1}, t_{2}\right]$ of the radiation. Because we are interested in the stationary correlation function which depends only on the difference $t_{2}-t_{1}=\tau$ one finally gets the following equation for $K_{A}[\tau]$ :

$$
\tau_{0} \frac{d K_{A}[\tau]}{d \tau}+K_{A}[\tau]=\frac{1}{N^{2}}\left[N K_{\sin }[\tau]+N(N-1)\langle\sin (\theta)\rangle^{2}\right],
$$

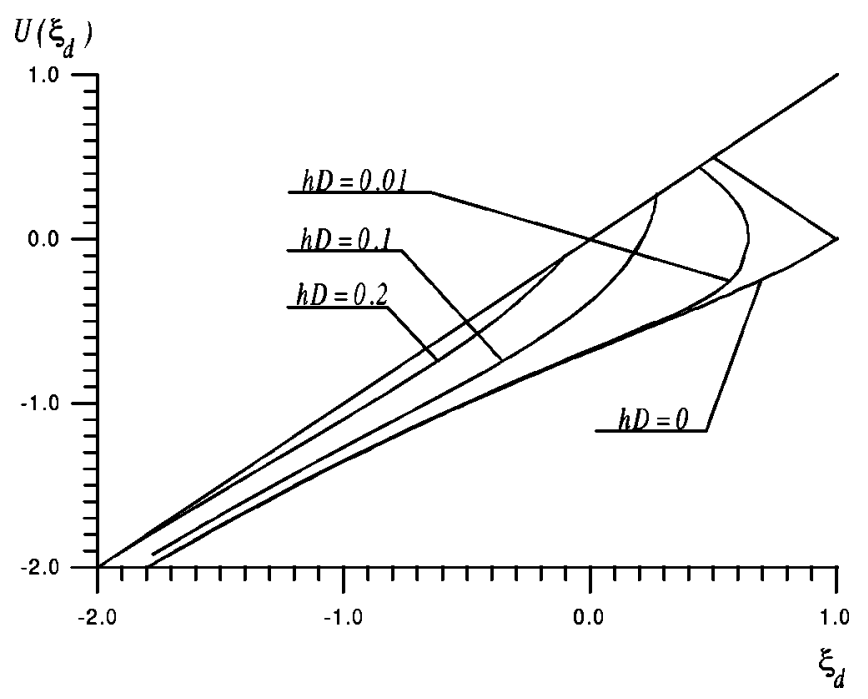

FIG. 4. Current-voltage characteristic calculated for different values of the noise intensity $h D$, with fixed $h \Gamma=1$. Compare to Fig. 2 and note the different scales. due to the fact that $\theta_{n}(t)$ and $\theta_{m}(t), n \neq m$, are uncorrelated. Here $\tau_{0}=1 / \Gamma$. In order to find the correlation function $K_{A}[\tau]$ one must know the correlation function $K_{\sin }[\tau]$ $=\left\langle\sin \left[\theta\left(t_{1}\right)\right] \sin \left[\theta\left(t_{1}+\tau\right)\right]\right\rangle, t_{1} \rightarrow \infty$.

By definition $K_{\sin }[\tau]$ is

$$
\begin{aligned}
K_{\sin }[\tau]= & \int_{-\pi}^{\pi} \sin \left(\theta_{0}\right) W_{r s t}\left(\theta_{0}\right) \int_{-\pi}^{\pi} \sin (\theta) \\
& \times W_{r}\left(\theta, \tau ; \theta_{0}, 0\right) d \theta d \theta_{0} .
\end{aligned}
$$

Here $W_{r}\left(\theta, \tau ; \theta_{0}, 0\right)$ is the reduced conditional probability density which is simply the probability density $W_{r}(\theta, t)$ obtained for a delta-shaped initial distribution Eq. (24). To obtain $K_{\sin }[\tau]$ we apply the new approach for description of stochastic processes. $^{25-27}$

First, let us define the correlation time $\tau_{\sin }$ as

$$
\tau_{\sin }=\frac{\int_{0}^{\infty}\left[K_{\sin }[\tau]-K_{\sin }[\infty]\right] d \tau}{K_{\sin }[0]-K_{\sin }[\infty]} .
$$

If the function $K_{\sin }[\tau]$ evolves exponentially in time $K_{\sin }[\tau] \sim e^{-\tau / \tau_{\sin }}$ then the time scale $\tau_{\sin }$ in the factor of the exponent coincides with the one defined by Eq. (32).

In order to find this correlation time we first need to introduce the mean time $\tau_{m}\left(\theta_{0}\right)$ of evolution of $\langle\sin (\theta)\rangle$ $=m_{\sin }(t)=\int_{-\pi}^{\pi} \sin (\theta) W_{r}(\theta, t) d \theta$

$$
\tau_{m}\left(\theta_{0}\right)=\frac{\int_{0}^{\infty}\left[m_{\sin }(t)-m_{\sin }(\infty)\right] d t}{m_{\sin }(0)-m_{\sin }(\infty)} .
$$

This time scale depends on the coordinate of the deltashaped initial distribution $\theta_{0}$. It is seen that the correlation time $\tau_{\text {sin }}$ on the basis of the definitions (31), (32), and (33), may be expressed via $\tau_{m}\left(\theta_{0}\right)$

$$
\tau_{\sin }=\frac{\int_{-\pi}^{\pi} \sin \left(\theta_{0}\right) W_{r s t}\left(\theta_{0}\right) \tau_{m}\left(\theta_{0}\right)\left[m_{\sin }(0)-m_{\sin }(\infty)\right] d \theta_{0}}{K_{\sin }[0]-K_{\sin }[\infty]}
$$

The time scale $\tau_{m}\left(\theta_{0}\right)$ may be found using the approach by Malakhov. ${ }^{25}$ We skip here some very long calculations and refer the interested reader to Ref. 27 where the time scale of evolution of average was obtained for another type of boundary conditions. Here we present only the final expression for $\tau_{m}\left(\theta_{0}\right)$

$$
\begin{aligned}
\tau_{m}\left(\theta_{0}\right)= & \frac{1}{D\left[m_{\sin }(0)-m_{\sin }(\infty)\right]}\left\{\int_{\theta_{0}}^{\pi}\left[C_{1}-\sin (x)\right]\right. \\
& \times e^{-u(x)} \int_{\theta_{0}}^{x} e^{u(y)} d y d x+\int_{-\pi}^{\pi}\left[\sin (x)-C_{1}\right] \\
& \left.\times e^{-u(x)} \int_{-\pi}^{x} e^{u(y)} \int_{-\pi}^{y} W_{r s t}(v) d v d y d x\right\},
\end{aligned}
$$

where $W_{r s t}(v)$ is expressed by Eq. (26) and 


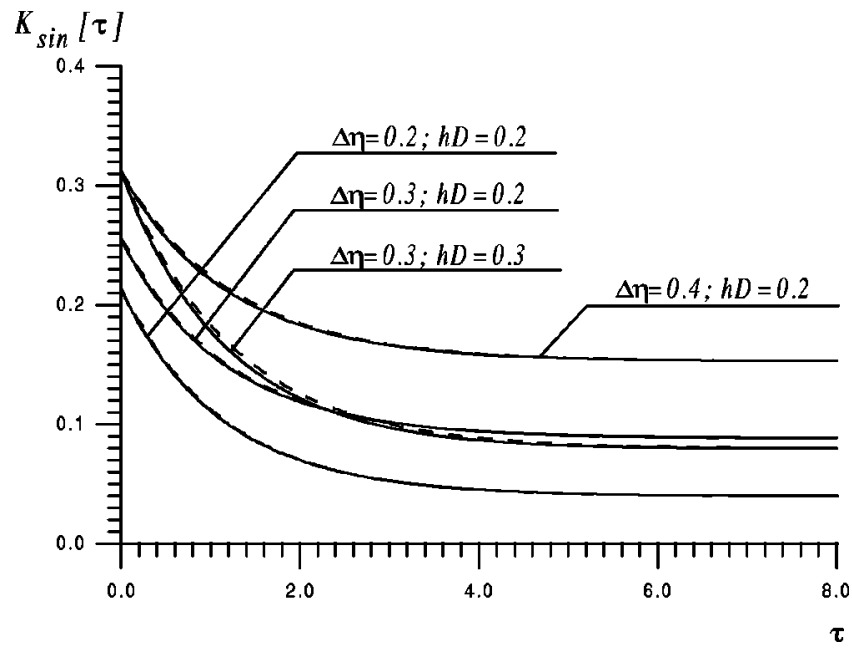

FIG. 5. Comparison between the exponential approximation (dashed line) and results of computer simulation (solid line) of the correlation function $K_{\sin }[\tau]$ for different values of $h D$ and $\Delta \eta$. See text.

$$
C_{1}=\int_{-\pi}^{\pi} \sin (x) e^{-u(x)} d x / \int_{-\pi}^{\pi} e^{-u(x)} d x
$$

Substituting $\tau_{m}\left(\theta_{0}\right)$ Eq. (35) into Eq. (34) one finds the correlation time $\tau_{\text {sin }}$

$$
\begin{aligned}
\tau_{\sin }= & \frac{h}{h D\left(K_{\sin }[0]-K_{\sin }[\infty]\right)}\left\{\int_{-\pi}^{\pi} \sin \left(\theta_{0}\right) W_{r s t}\left(\theta_{0}\right)\right. \\
& \times \int_{\theta_{0}}^{\pi}\left[C_{1}-\sin (x)\right] e^{-u(x)} \int_{\theta_{0}}^{x} e^{u(y)} d y d x d \theta_{0} \\
& +\int_{-\pi}^{\pi} \sin \left(\theta_{0}\right) W_{r s t}\left(\theta_{0}\right) d \theta_{0} \int_{-\pi}^{\pi}\left[\sin (x)-C_{1}\right] \\
& \left.\times e^{-u(x)} \int_{-\pi}^{x} e^{u(y)} \int_{-\pi}^{y} W_{r s t}(v) d v d y d x\right\},
\end{aligned}
$$

where $\quad K_{\sin }[0]=\int_{-\pi}^{\pi} \sin ^{2}(x) W_{r s t}(x) d x, \quad K_{\sin }[\infty]=\langle\sin (x)\rangle^{2}$, and $\langle\sin (x)\rangle=\int_{-\pi}^{\pi} \sin (x) W_{r s t}(x) d x$. In this formula we put $h$ both in the numerator and the denominator because later we shall see that $\tau_{\sin } \sim h$.

Once we know the time scale of the evolution of the correlation function we can present it in the form

$$
K_{\sin }[\tau]=\left(K_{\sin }[0]-K_{\sin }[\infty]\right) e^{-\tau / \tau_{\sin }}+K_{\sin }[\infty]
$$

where $\tau_{\text {sin }}$ is given by Eq. (36). Our assumption about the exponential behavior of the correlation function is based on previous investigations ${ }^{26,27}$ and is confirmed by computer simulations.

The exponential approximation, Eq. (37), and the results of computer simulation of the correlation function $K_{\sin }[\tau]$ are shown in Fig. 5. First, it is seen, that the exponential approximation agrees well even in the limit where the equivalent noise intensity $h D$ is rather large compared with the barrier height that separates the stable states of the potential Eq. (23). Second, and important, is that the correlation

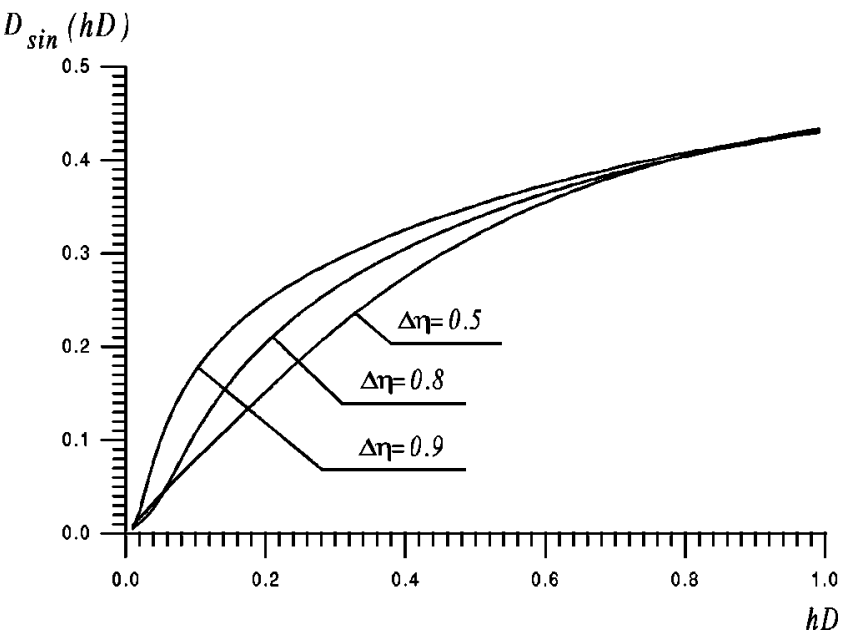

FIG. 6. The calculated variance, $D_{\sin }=K_{\sin }[0]-K_{\sin }[\infty]$, as function of noise intensity $h D$ for three bias current values, $\Delta \eta$ $=0.5,0.8$, and 0.9. See text.

time $\tau_{\text {sin }}$ is insensitive to variations of $h D$ and $\tau_{\sin }=h C$, where $C$ is a numeric coefficient ranging from about $0.8-1.5$; usually $C \approx 1.2$.

Thus, Eq. (30) can be solved analytically with a solution of the following form:

$$
\begin{aligned}
K_{A}[\tau]= & \left\{K_{\sin }[\infty]+\frac{\left(K_{\sin }[0]-K_{\sin }[\infty]\right)}{N\left(\tau_{\sin }-\tau_{0}\right)}\right. \\
& \times\left[\tau_{\sin } e^{\left.-\tau / \tau_{\sin }-\tau_{0} e^{-\tau / \tau_{0}}\right]}\right\} .
\end{aligned}
$$

This is the correlation function of the amplitude $A^{\prime}$ $=A \Gamma / \epsilon N$ and to get the correlation function for $A$ the righthand side of Eq. (38) should be multiplied by $(N \epsilon / \Gamma)^{2}$.

Now, knowing the correlation function of the amplitude, we can find the variance of the amplitude $D_{A}$, which we supposed to be small; $\left\langle A^{\prime}\right\rangle \gg \sqrt{\left\langle A^{\prime 2}\right\rangle-\left\langle A^{\prime}\right\rangle^{2}}$. By definition we have $K_{A}[0]=\left\langle A^{\prime 2}\right\rangle, K_{A}[\infty]=\left\langle A^{\prime}\right\rangle^{2}$. Also by the definition $D_{A}=\left\langle A^{\prime 2}\right\rangle-\left\langle A^{\prime}\right\rangle^{2}$, so the variance has the following form

$$
D_{A}=\frac{\left(K_{\sin }[0]-K_{\sin }[\infty]\right)}{N} .
$$

It is seen that the variance of the amplitude is proportional to the variance of $\sin \theta_{n}, D_{\sin }=K_{\sin }[0]-K_{\sin }[\infty]$ which decreases with the noise intensity, $h D$ (see Fig. 6) and is inversely proportional to the number of fluxons emitting the wave. If we reduce the amplitude $\left\langle A^{\prime}\right\rangle$ in the potential, Eq. (23), e.g., corresponding to a reduced coupling, we observe that the variance $D_{\sin }$ increases and reaches 0.5 for $\left\langle A^{\prime}\right\rangle$ $=0$. Accordingly, the collective effect of the Cherenkov synchronism of fluxons with the emitted wave improves the noise properties of the CFFO.

Finally, the spectrum $S_{A}(\omega)=\int_{0}^{+\infty} K_{A}[\tau] \cos (\omega \tau) d \tau$ may be obtained as the Fourier transformation of the correlation function $K_{A}[\tau]$ and represents a sum of two Lorentzians 


$$
\begin{aligned}
S_{A}(\omega)= & \left\{\frac{\left(K_{\sin }[0]-K_{\sin }[\infty]\right)}{N\left(\tau_{\sin }-\tau_{0}\right)}\left[\frac{2}{\left(1 / \tau_{\sin }^{2}+\omega^{2}\right)}-\frac{2}{\left(1 / \tau_{0}^{2}+\omega^{2}\right)}\right]\right. \\
& +K_{\sin [\infty] \delta(\omega)}[.
\end{aligned}
$$

The generation frequency equals $\Omega_{0}=\omega_{0}+\langle\dot{\chi}\rangle$ and we will consider fluctuations near $\Omega_{0} ; \nu(t)=\dot{\chi}-\langle\dot{\chi}\rangle$. The correlation function $K_{\nu}[\tau]$ and the spectrum $S_{\nu}[\omega]$ may be obtained in the same way as the corresponding characteristics of the amplitude. From Eq. (21) one can get

$$
K_{\nu}[\tau]=\left(\frac{\Gamma}{\left\langle A^{\prime}\right\rangle}\right)^{2} \frac{\left(K_{\cos }[0]-K_{\cos }[\infty]\right)}{N} e^{-\tau / \tau_{\cos }},
$$

where $K_{\cos }[0]=\int_{-\pi}^{\pi} \cos ^{2}(x) W_{r s t}(x) d x, \quad K_{\cos }[\infty]=\langle\cos (x)\rangle^{2}$, and $\langle\cos (x)\rangle=\int_{-\pi}^{\pi} \cos (x) W_{r s t}(x) d x$. The correlation time $\tau_{\cos }$ has the following form:

$$
\begin{aligned}
\tau_{\cos }= & \frac{h}{h D\left(K_{\cos [}[0]-K_{\cos [}[\infty]\right)}\left\{\int_{-\pi}^{\pi} \cos \left(\theta_{0}\right) W_{r s t}\left(\theta_{0}\right)\right. \\
& \times \int_{\theta_{0}}^{\pi}\left[C_{2}-\cos (x)\right] e^{-u(x)} \int_{\theta_{0}}^{x} e^{u(y)} d y d x d \theta_{0} \\
& +\int_{-\pi}^{\pi} \cos \left(\theta_{0}\right) W_{r s t}\left(\theta_{0}\right) d \theta_{0} \int_{-\pi}^{\pi}\left[\cos (x)-C_{2}\right] \\
& \left.\times e^{-u(x)} \int_{-\pi}^{x} e^{u(y)} \int_{-\pi}^{y} W_{r s t}(v) d v d y d x\right\},
\end{aligned}
$$

where $C_{2}=\int_{-\pi}^{\pi} \cos (x) e^{-u(x)} d x / \int_{-\pi}^{\pi} e^{-u(x)} d x$. It is seen, that the noise properties of the frequency deviations are similar to what is found for the amplitude

$$
D_{\nu}=\frac{1}{N}\left(\frac{\Gamma}{\left\langle A^{\prime}\right\rangle}\right)^{2}\left(K_{\mathrm{cos} L}[0]-K_{\cos }[\infty]\right),
$$

i.e., the variance of the frequency decreases with decreasing noise intensity $h D$, mode damping rate $\Gamma$, and with increasing number of fluxons $N$. The correlation time $\tau_{\text {cos }}$, also similar to $\tau_{\sin }$, has the form $\tau_{\cos }=h C$, where $C$ is of the order unity.

The spectrum of the frequency deviations is given by

$$
S_{\nu}(\omega)=\left(\frac{\Gamma}{\left\langle A^{\prime}\right\rangle}\right)^{2}\left\{\frac{\left(K_{\cos }[0]-K_{\cos }[\infty]\right)}{N \tau_{\cos }}\left[\frac{2}{\left(1 / \tau_{\cos }^{2}+\omega^{2}\right)}\right]\right\} \text {. }
$$

Now, when we know the basic characteristics of the amplitude and the frequency of the CFFO, namely, their correlation functions and spectra, we can perform the final analysis of the oscillator linewidth using standard procedures.

\section{RADIATION LINEWIDTH OF THE CFFO}

The theoretical methods for spectral analysis of oscillators are well established (see, e.g., Ref. 28). In the most general form the task may be formulated as follows; an oscillator has an output $z(t)=A(t) \cos [\omega t+\chi(t)]$. The aim is to determine the spectrum of this signal if the statistical characteristics of $A(t)$ and $\chi(t)$ or $\dot{\chi}(t)$ are known.

In the general case when the fluctuations of $A(t)$ and $\dot{\chi}(t)$ are correlated, the analysis is possible only when the fluctuations of amplitude are small and the correlation function $K_{A \nu}[\tau]$ is of the order or less than $K_{A}[\tau]$. Here we are working in the limit of small amplitude fluctuations so using the approach of the previous section it can be shown that the spectrum $K_{A \nu}[\tau]$ is less than $K_{A}[\tau]$. Following Malakhov ${ }^{28}$ the spectrum of the emitted wave $z(t)=A(t) \cos [\omega t+\chi(t)]$ consists of a narrow, slightly asymmetric peak centered near the radiation frequency $\Omega_{0}$ due to the frequency fluctuations and a small, but broad, asymmetric pedestal originating from the amplitude fluctuations and the correlated amplitudefrequency fluctuations.

Let us analyze the spectral peak and its spectral width (linewidth of the emitted signal). According to Malakhov ${ }^{28}$ a detailed analysis of the spectral form $W_{z}(\Omega)$ and the linewidth $\Delta \Omega$ may be done only for stationary frequency fluctuations having a normal distribution. This is fulfilled here. In addition due to the central limit theorem the condition of a Gaussian distribution of the frequency fluctuations is fulfilled for a large number of fluxons $N$, because the random process in Eq. (21) is a sum of a large number of nearly equal components. In this case the form of a spectral peak $W_{z}(\Omega)$ is

$$
W_{z}(\Omega)=\frac{\langle A\rangle^{2}}{4 \pi} \int_{-\infty}^{+\infty} \exp \left[-\int_{0}^{\tau}(\tau-\xi) K_{\nu}[\xi] d \xi\right] \cos \Omega \tau d \tau .
$$

As usual [see Ref. 28 and Eq. (32)] the spectral width of the frequency fluctuations is defined as

$$
\nu_{0}=\frac{K_{\nu}[0]}{\int_{0}^{\infty} K_{\nu}[\tau] d \tau}=\frac{1}{\pi} \frac{D_{\nu}}{S_{\nu}(0)}=1 / \tau_{\cos } .
$$

Let us introduce the modulation index

$$
m=D_{\nu} \tau_{\cos }^{2}=\frac{\pi S_{\nu}(0)}{\nu_{0}} .
$$

For the particular case of an exponential correlation function for the frequency, Eq. (41), one can find an exact expression for the shape of the spectral line for arbitrary values of $m$

$$
\begin{aligned}
W_{z}(\Omega) & =\frac{\langle A\rangle^{2}}{2 \pi} e^{m} \int_{0}^{\infty} \exp \left[m\left(-\nu_{0} \tau-e^{-\nu_{0} \tau}\right)\right] \cos \Omega \tau d \tau \\
& =\frac{\langle A\rangle^{2}}{2 \pi} e^{m} \sum_{\lambda=0}^{\infty} \frac{(-m)^{\lambda}}{\lambda !} \frac{\nu_{0}(m+\lambda)}{\nu_{0}^{2}(m+\lambda)^{2}+\Omega^{2}} .
\end{aligned}
$$

For $m \ll 1$ the expression for $W_{z}(\Omega)$ is (see Ref. 28)

$$
W_{z}(\Omega)=\frac{\langle A\rangle^{2}}{2 \pi} \frac{m \nu_{0}^{3}}{\left[\nu_{0}^{2} m^{2}+\Omega^{2}\right]\left[\nu_{0}^{2}+\Omega^{2}\right]} .
$$

In the opposite case $m \gg 1$ the shape of the spectral line has a Doppler (or Gaussian) form 


$$
W_{z}(\Omega)=\frac{\langle A\rangle^{2}}{2} \frac{1}{\sqrt{2 \pi D_{\nu}}} e^{-\Omega^{2} /\left(2 D_{\nu}\right)} .
$$

The spectral linewidth $\Delta \Omega$ for arbitrary $m$ may be written as

$$
\Delta \Omega=\pi m \nu_{0} B(m), \quad B(m)=\frac{e^{-m}}{\sum_{\lambda=0}^{\infty} \frac{(-m)^{\lambda}}{\lambda !} \frac{m}{(m+\lambda)} .}
$$

For $m \ll 1$

$$
B(m)=1-m+0.5 m^{2}-0.416 m^{3}+\cdots
$$

and, therefore,

$$
\Delta \Omega=\pi m \nu_{0}, \quad m \ll 1 .
$$

For $m \gg 1$

$$
\begin{gathered}
B(m)=\sqrt{2 \pi / m} M(m), \\
M(m)=1-0.266 \frac{1}{\sqrt{m}}+0.084 \frac{1}{m}+0.153 \frac{1}{m \sqrt{m}}+\cdots,
\end{gathered}
$$

and the linewidth has the form $\Delta \Omega=\sqrt{2 \pi D_{\nu}} M(m)$. Notice that (see Ref. 28) for $m<0.1$ one can use the expansion Eq. (50) and for $m>10$ the expansion Eq. (52). For $0.1<m$ $<10 B(m)$ varies from approximately 0.9 for $m=0.1$ to 0.3 for $m=10$.

The experimental parameters ${ }^{2,3}$ show that a small modulation index, $m \ll 1$, is more realistic for practical CFFOs and in this case the radiation linewidth, Eq. (51), takes the form

$$
\begin{aligned}
\Delta \Omega & =\frac{\pi h C \Gamma^{2}}{N} \Delta \Omega^{*} \\
& =\frac{2 \Gamma \alpha_{1} C}{k_{r}^{2} l \sqrt{1-\omega_{r}^{2} / k_{r}^{2}}} \operatorname{Re}\left(\left.\frac{\partial D\left(\omega, k_{r}\right)}{\partial \omega}\right|_{\omega_{0}}\right)^{-1} \frac{D_{\text {cos }}}{\langle A\rangle^{2}}, \quad m \ll 1,
\end{aligned}
$$

where

$$
\Delta \Omega^{*}=D_{\cos } /\left\langle A^{\prime}\right\rangle^{2},
$$

and $D_{\cos }=K_{\cos }[0]-K_{\cos }[\infty]=\left\langle\cos (\theta)^{2}\right\rangle-\langle\cos (\theta)\rangle^{2} . C$ is of the order of unity. Thus, the radiation linewidth is proportional to the damping coefficients of the wave $(\Gamma)$ and the junction $\left(\alpha_{1}\right)$, and is inversely proportional to the emitted power $\langle A\rangle^{2}$ and the wave number $k_{r}^{2}$. The variance $D_{\text {cos }}(h D)$ is a nonlinear function of the noise intensity $D$ and the parameter $h=\Gamma \alpha_{1} / \sigma \epsilon N$.

Taking $\alpha_{1}=0.1, h=100, N=30$, and $h \Gamma=0.01 ; 0.1 ; 1$ (the corresponding IVCs were shown in Figs. 3 and 4), one finds $\Delta \Omega=10^{-7} \Delta \Omega^{*}, \quad \Delta \Omega=10^{-5} \Delta \Omega^{*}, \quad$ and $\Delta \Omega$ $=10^{-3} \Delta \Omega^{*}$, respectively. In Eq. (49) and Eqs. (51) $-(53)$ the linewidth, $\Delta \Omega$, is expressed in units of the maximal Josephson plasma frequency. For the usual FFO (Ref. 29) the linewidth is $10^{-6}-10^{-8}$ times the oscillator frequency. For

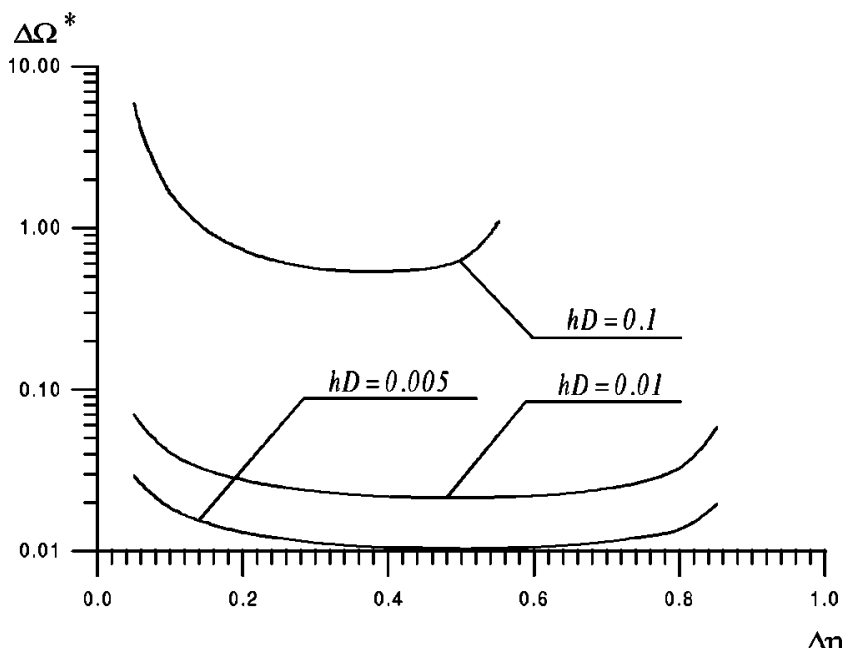

FIG. 7. Linewidth of the emitted radiation $\Delta \Omega^{*}$ as function of bias current for three values of the noise intensity $h D$. The zero on horizontal axis is shifted compared to Figs. 1-3 [see Eq. (23)]. All parameters are normalized, see text.

the CFFO the linewidth contains the factor $\Delta \Omega^{*}$ which is smaller than unity and decreases to zero with decreasing noise intensity as demonstrated in Fig. 7 for $h \Gamma=0.1$, and $D=10^{-3}, D=10^{-4}$, and $D=0.5 \times 10^{-4}$. From the plot of $\Delta \Omega^{*}$, Eq. (54), versus bias current $\Delta \eta$, Eq. (23), one observes an interesting phenomenon. The linewidth has a minimum at a certain bias current which is slightly different from the value yielding the maximal amplitude of generation. In summary, because of resonant character of the wave-fluxon interaction, and substantiated by the estimations presented above, the CFFO will have a significantly smaller linewidth than the usual FFO.

\section{CONCLUSIONS}

In the present paper the influence of thermal fluctuations on the Cherenkov radiation effect in a long annular Josephson junction with an overlaying transmission line has been investigated. It is shown that the emitted radiation is reduced in the presence of noise. The physical explanation is that without noise the maximal output power is obtained in the synchronous regime when the fluxons bunch in the strongest "slowing" field of the electromagnetic wave. In this critical point of operation any additional increase of the bias current (e.g., also due to fluctuations) will destroy the state where the fluxon is trapped by the wave. So, at the bias current which corresponds to the maximal output power without noise the synchronism between the fluxons and the wave is unstable with respect to fluctuations. This effect was considered within the framework of a rather simple but still realistic model based on an annular Cherenkov flux flow oscillator (CFFO). It is believed that this is valid also for a CFFO with linear geometry.

Further it has been shown that the influence of the radiated wave on the fluxon dynamics leads to several new and interesting phenomenon. Both the mean amplitude of the emitted radiation and its linewidth exhibits a resonant behavior as functions of junction bias current at a given noise intensity. The found formulas for the radiation spectrum of 
the CFFO predict a smaller radiation linewidth and a higher emitted power than the usual flux flow oscillator (FFO).

For low noise intensity and relatively week coupling between the junction and the slow wave transmission line the obtained theoretical results allow for an optimization of the main CFFO parameters (power, tunability, and bandwidth). In the limit the obtained analytical results are believed to enable practical design and realization of oscillators based on Cherenkov radiation of Josephson fluxons. Numerical simulations will be needed to explore the properties of future CFFOs operating in the limit of strong coupling and with more complex geometries.

The oscillator based on the Cherenkov radiation of the fluxons in Josephson systems is a new and promising source of radiation. For applications as local oscillator for SIS mixer based $\mathrm{mm}$ - and sub-mm wave receivers the CFFO is particularly attractive especially at frequencies above $\approx 1 / 3$ of the superconducting gap frequency where the linewidth of the usual $\mathrm{Nb} / \mathrm{AlOx} / \mathrm{Al}$ FFO increases due to higher damping caused by self-excitation of quasiparticles. Also surface losses start to play a crucial role at such high frequencies. Similar to the FFO the CFFO can undoubtedly be phase locked to an external reference oscillator. This, however, still needs experimental verification. Finally, the CFFO concept appears very promising for oscillators fabricated with high$T_{c}$ superconductors.

\section{ACKNOWLEDGMENTS}

The authors wish to thank V. V. Kurin for constructive comments to the manuscript and A. N. Malakhov and V. A. Markelov for helpful discussions. This work has been supported by the Russian Foundation for Basic Research (Project No. 96-15-96718, Project No. 97-02-16928, and Project No. 99-02-17544), Ministry of High Education of Russian Federation (Project No. 3877), Grant No. 98-2-13 from the International Center for Advanced Studies in Nizhny Novgorod, the Danish Natural Science Council, and the Danish Research Academy.
${ }^{1}$ Yu. S. Kivshar and B. A. Malomed, Phys. Rev. B 37, 9325 (1988); Rev. Mod. Phys. 61, 763 (1989).

${ }^{2}$ A. M. Baryshev, A. V. Yulin, V. V. Kurin, V. P. Koshelets, S. V. Shitov, A. V. Shchukin, P. N. Dmitriev, and L. V. Filippenko, IEEE Trans. Appl. Supercond. 9, 3737 (1999).

${ }^{3}$ V. V. Kurin, A. Yulin, E. Goldobin, A. Klushin, H. Kohlstedt, M. Levitchev, and N. Thyssen, IEEE Trans. Appl. Supercond. 9, 3733 (1999).

${ }^{4}$ R. G. Mints and I. B. Snapiro, Phys. Rev. B 52, 9691 (1995).

${ }^{5}$ S. N. Artemenko and S. V. Remisiov, Pis'ma Zh. Éksp. Teor. Fiz. 66, 811 (1997) [JETP Lett. 66, 853 (1997)].

${ }^{6}$ V. V. Kurin, A. V. Yulin, I. A. Shereshevsky, and N. K. Vdovicheva, Phys. Rev. Lett. 80, 3372 (1998).

${ }^{7}$ J. G. Caputo, N. Flytzanis, N. Lazarides, V. V. Kurin, and A. Chiginev (unpublished)

${ }^{8}$ H. S. J. van der Zant, T. P. Orlando, S. Watanabe, and S. H. Strogatz, Phys. Rev. Lett. 74, 174 (1995).

${ }^{9}$ G. Hechtfisher, R. Kleiner, A. V. Ustinov, and P. Mueller, Phys. Rev. Lett. 79, 1365 (1997).

${ }^{10}$ T. Nagatsuma, K. Enpuku, F. Irie, and K. Yoshida, J. Appl. Phys. 54, 3302 (1983); 56, 3284 (1984); 58, 441 (1985); 63, 1130 (1988).

${ }^{11}$ E. Goldobin, A. Wallraff, N. Thyssen, and A. V. Ustinov, Phys. Rev. B 57, 130 (1998).

${ }^{12}$ D. W. McLaughlin and A. C. Scott, Phys. Rev. A 18, 1652 (1978).

${ }^{13}$ E. Jørgensen, V. P. Koshelets, R. Monaco, J. Mygind, M. R. Samuelsen, and M. Salerno, Phys. Rev. Lett. 49, 1093 (1982).

${ }^{14}$ V. P. Koshelets, S. V. Shitov, A. V. Shchukin, L. V. Filippenko, P. N. Dmitriev, V. L. Vaks, J. Mygind, A. B. Baryshev, W.
Luinge, and H. Golstein, IEEE Trans. Appl. Supercond. 9, 4133 (1999).

${ }^{15}$ J. Mygind, V. P. Koshelets, S. V. Shitov, L. V. Filippenko, V. L. Vaks, A. M. Baryshev, W. Luinge, and N. Whyborn, Supercond. Sci. Technol. 12, 720 (1999).

${ }^{16}$ V. V. Kurin and A. V. Yulin, Phys. Rev. B 55, 11659 (1997).

${ }^{17}$ A. M. Baryshev, A. V. Yulin, V. V. Kurin, V. P. Koshelets, P. N. Dmitriev, and L. V. Filippenko, Supercond. Sci. Technol. 12, 967 (1999).

${ }^{18}$ N. Martucciello, J. Mygind, V. Koshelets, A. Shchukin, L. Fillipenko, and R. Monaco, Phys. Rev. B 57, 5444 (1998).

${ }^{19}$ M. B. Mineev, G. S. Mkrtchyan, and V. V. Smidt, J. Low Temp. Phys. 45, 497 (1981).

${ }^{20}$ T. Holst, J. B. Hansen, N. Grønbech-Jensen, and J. A. Blackburn, Phys. Rev. B 42, 127 (1990).

${ }^{21}$ R. L. Stratonovich, Topics of the Theory of Random Noise (Gordon and Breach, New York, 1963).

${ }^{22}$ P. A. Lee, J. Appl. Phys. 42, 325 (1971).

${ }^{23}$ V. I. Tikhonov and M. A. Mironov, Markovian Processes (Sovetskoe Radio, Moscow, 1978) (in Russian).

${ }^{24}$ V. Ambegaokar and B. I. Halperin, Phys. Rev. Lett. 22, 1364 (1969).

${ }^{25}$ A. N. Malakhov, Chaos 7, 488 (1997).

${ }^{26}$ S. P. Nikitenkova and A. L. Pankratov, Phys. Rev. E 58, 6964 (1998).

${ }^{27}$ A. L. Pankratov, Phys. Lett. A 255, 17 (1999).

${ }^{28}$ A. N. Malakhov, Fluctuations in Autooscillating Systems (Science, Moscow, 1968) (in Russian).

${ }^{29}$ V. P. Koshelets, S. V. Shitov, A. V. Shchukin, L. V. Filippenko, J. Mygind, and A. V. Ustinov, Phys. Rev. B 56, 5572 (1997). 